\title{
3D Laser Scanner for Tunnel Surveying and Accuracy Analysis According to Registration Method
}

\author{
Joon Kyu Park ${ }^{1}$, Kap Yong Jung2* \\ ${ }^{1}$ Department of Civil Engineering, Seoil University, 28, Yongmasan-ro 90-gil, \\ Jungnang-gu, Seoul, Korea, \\ ${ }^{2}$ Department of Civil Engineering, Graduate School, Chungnam National University, \\ 99 Daehak-ro, Yuseong-gu, Daejeon, Korea, \\ 1'jkpark@seoil.ac.kr,2jungjusa@hanmail.net
}

\begin{abstract}
The tunnel is an important facility in the United States and Canada and is recognized as an infrastructure for eco-friendly urban construction among the structures that make up smart cities. Total Station Measurements and Global Navigation Satellite Systems (GNSS) have the disadvantage of long working time and impossible satellite reception for tunnel measurements. Recently, 3D laser scanners have been used in a variety of areas as a new way to improve existing surveying methods. In this study, $3 D$ laser scanners were used for tunnel measurements to assess its usefulness. Scan data was obtained by configuring traverse using the Total Station function and compared with check points at 10 points already installed for accuracy verification. Results of accuracy evaluation compared with check points, the maximum error was within $6 \mathrm{~cm}$ in the $N, E$, and $H$ directions, indicating the plane and elevation acceptable accuracy of scale 1:1000 digital maps, and suggesting the applicability of methods using reference point performance and laser scan data. Scanning data enables continuous analysis of scan section shapes as well as cross sectional analysis. Further research can improve the accuracy of the feature registration method, which can improve the tunnel survey efficiency.
\end{abstract}

Keywords: 3D Laser Scanner, Accuracy Analysis, Pointcloud, Registration, Tunnel Surveying

\section{Introduction}

Measurement equipment currently used at the tunnel construction site include GNSS, total stress and 3D laser scanners[1][2][3]. Total Station uses the phase difference of the laser reflected on the target to measure the distance, and calculates the coordinates of the target using the measured distance and the angle of the laser[4][5]. Total Station measurements take a long time to acquire data, and GNSS using satellites cannot be used because satellites cannot be received in tunnels[6]. Recently, 3D laser scanners have been seen as a device that complements the shortcomings of a small amount of data and long measurement time at the entire station. 3D laser scanners are used in a variety of areas. Research and application methods using 3D laser scanners are mainly used in civil engineering[7]. Total Station has been used mainly because GNSS is impossible to measure in tunnel measurements. Total Station has been used

Article history:

Received (February 13, 2019), Review Result (July 21, 2019), Accepted (September 11, 2019) 
to observe tunnels as three-dimensional coordinates and to compare and analyze the design values of various stages of construction[8][9]. As total station surveying takes a lot of time to acquire data, the use of 3D laser scanners for tunnel surveying is increasing recently. However, when using the 3D laser scanner, a large number of reference points must be installed inside the tunnel for registration and geo-referencing of acquired data. As an emerging technology, a shape matching method has been introduced in the field of scanning sensors, and its use is expected. In this study, tunnel survey using 3D laser scanner was performed and its utility was evaluated. And the accuracy of the shape registration method for recent registration was analyzed.

\section{Data acquisition}

In this study, the tunnel construction site in Yangpyeong was selected as the study area for tunnel surveying using scanning total stations, and data on some tunnels were obtained. Data on the $300 \mathrm{~m}$ extension of the highway tunnel section has been secured. The scanning total station used for data acquisition is the SX10 model, which integrates the total station and 3D laser scanner. Figure 3 shows the SX10. Figure 1 shows the study area and figure 2 shows SX10.

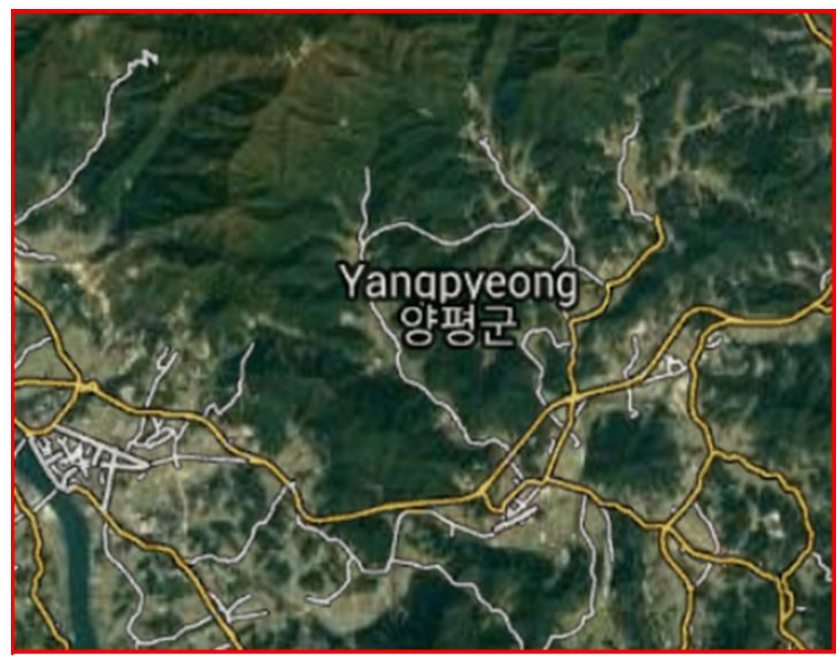

Figure. 1 Study area

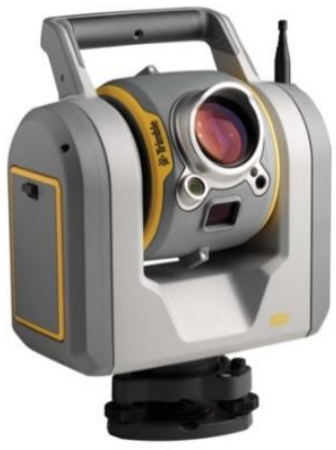

Figure. 2 SX10[10] 
Data collection began with the reference point of the tunnel entrance and moved the mechanical point into the tunnel. Considering redundancy for data processing, the scan was performed once every $100 \mathrm{~m}$ and data was acquired a total of five times with approximately $50 \%$ redundancy. The time required for data collection was less than 1 hour and 30 minutes compared to the entire station. Fig. 3 shows scanning data.

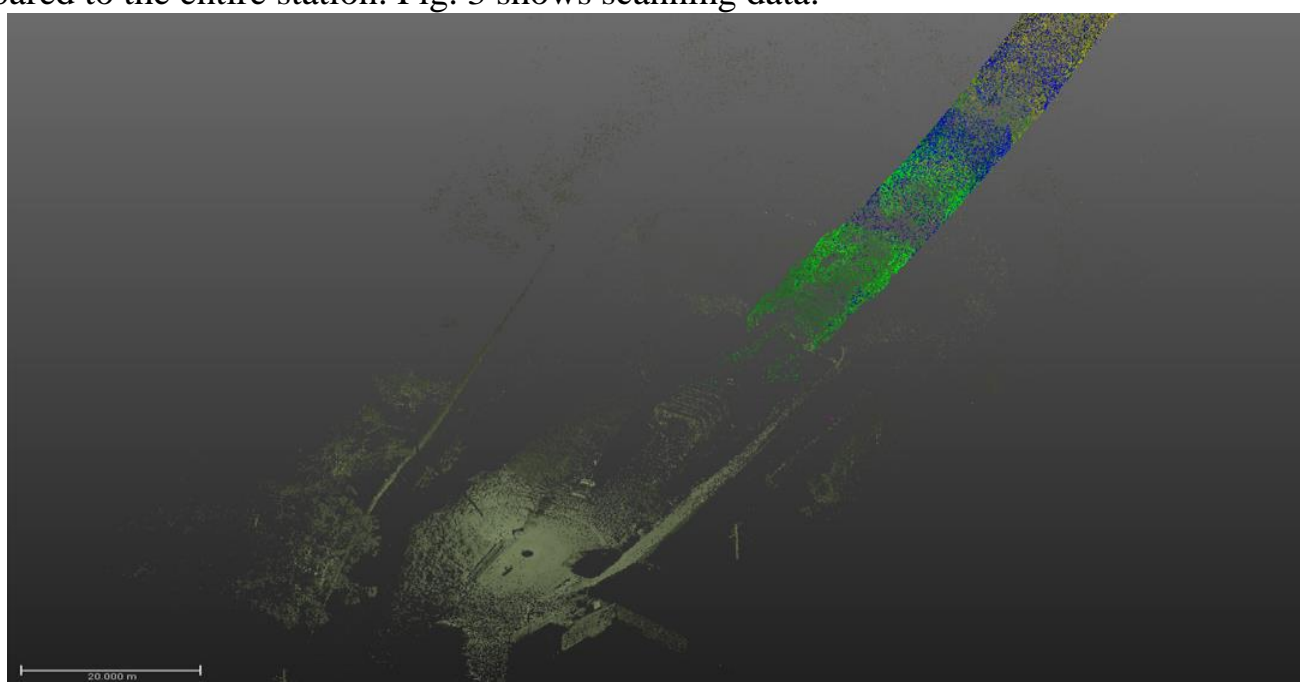

Figure. 3 Scanning data

The data was acquired by configuring the traverse using the total station function, and compared with the check points of the 10 points already installed for the accuracy verification.

\section{Data processing and analysis}

Data matching was performed in RealWorks software to process scan data, and comparisons in the design section were performed. Data matching was performed using the traverse function of the scan total station. Finally, the modelling results of the tunnel were created to compare with the design drawings. Figure 4 shows the results of the modelling.

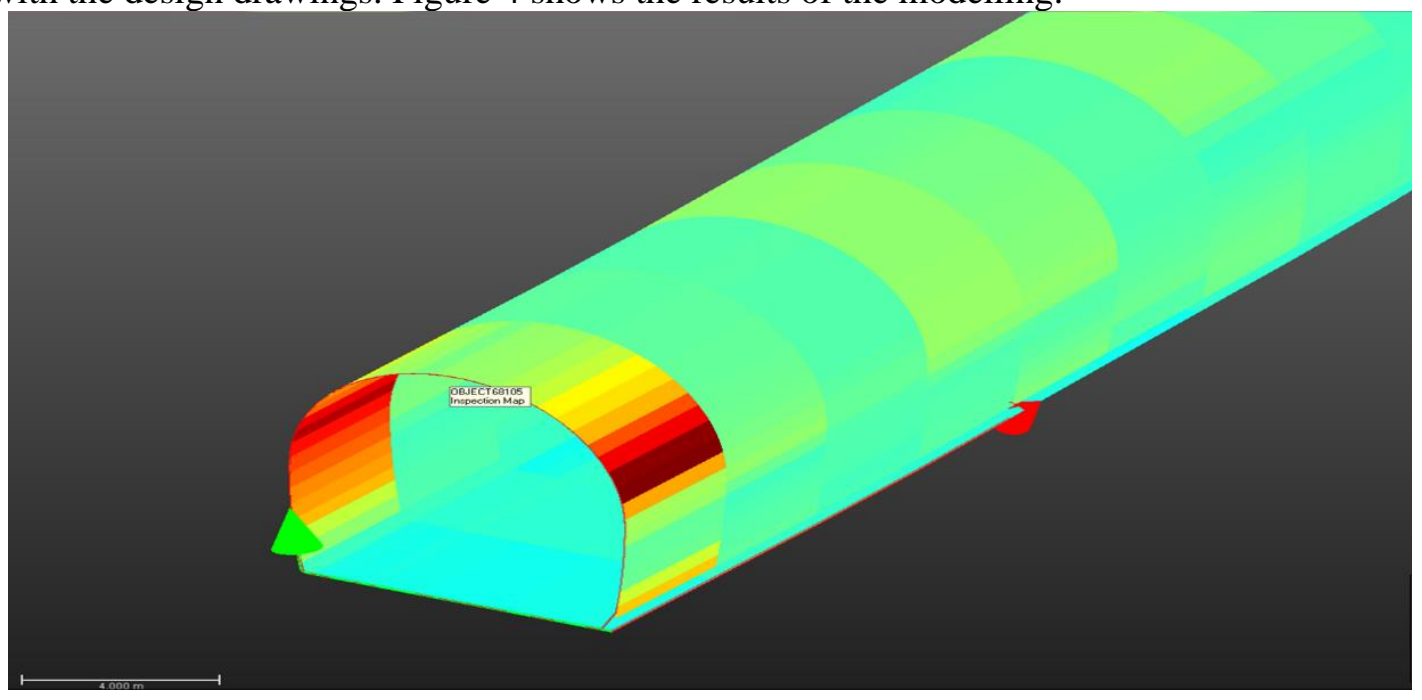

Figure. 4 Modeling result 
To evaluate the accuracy of the tunnel constructed with the 3D laser scanner, the performance of 10 test points was compared. Fig. 5 shows the comparison of accuracy of check points.

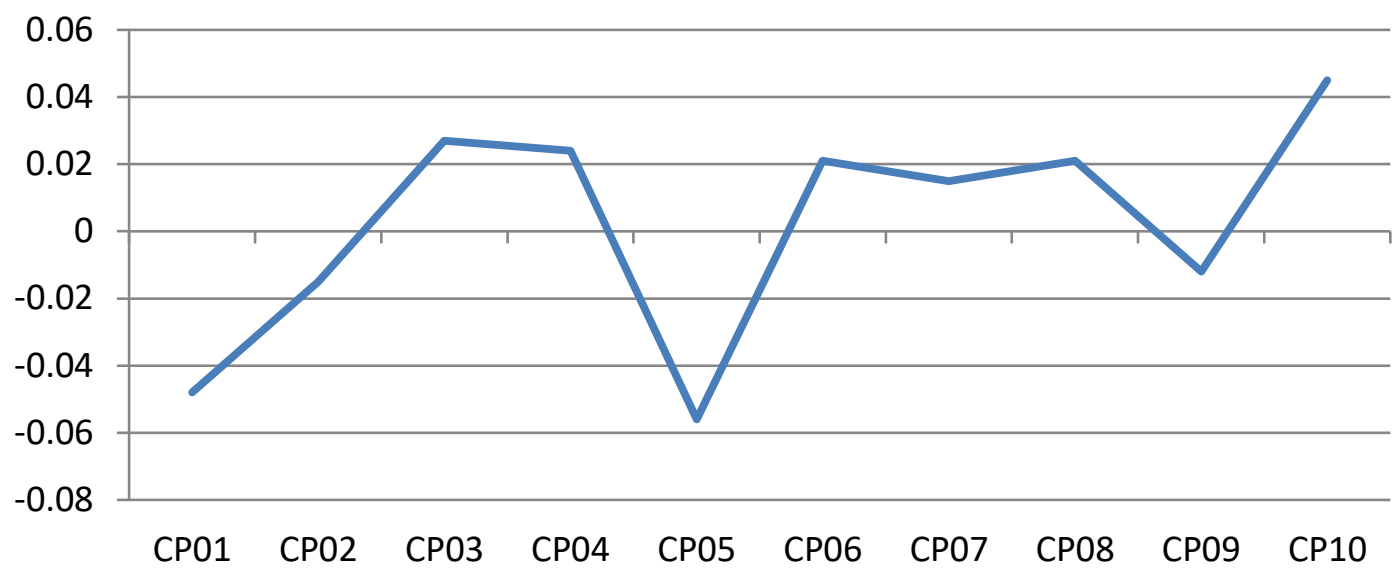

(a) $\mathrm{dX}$
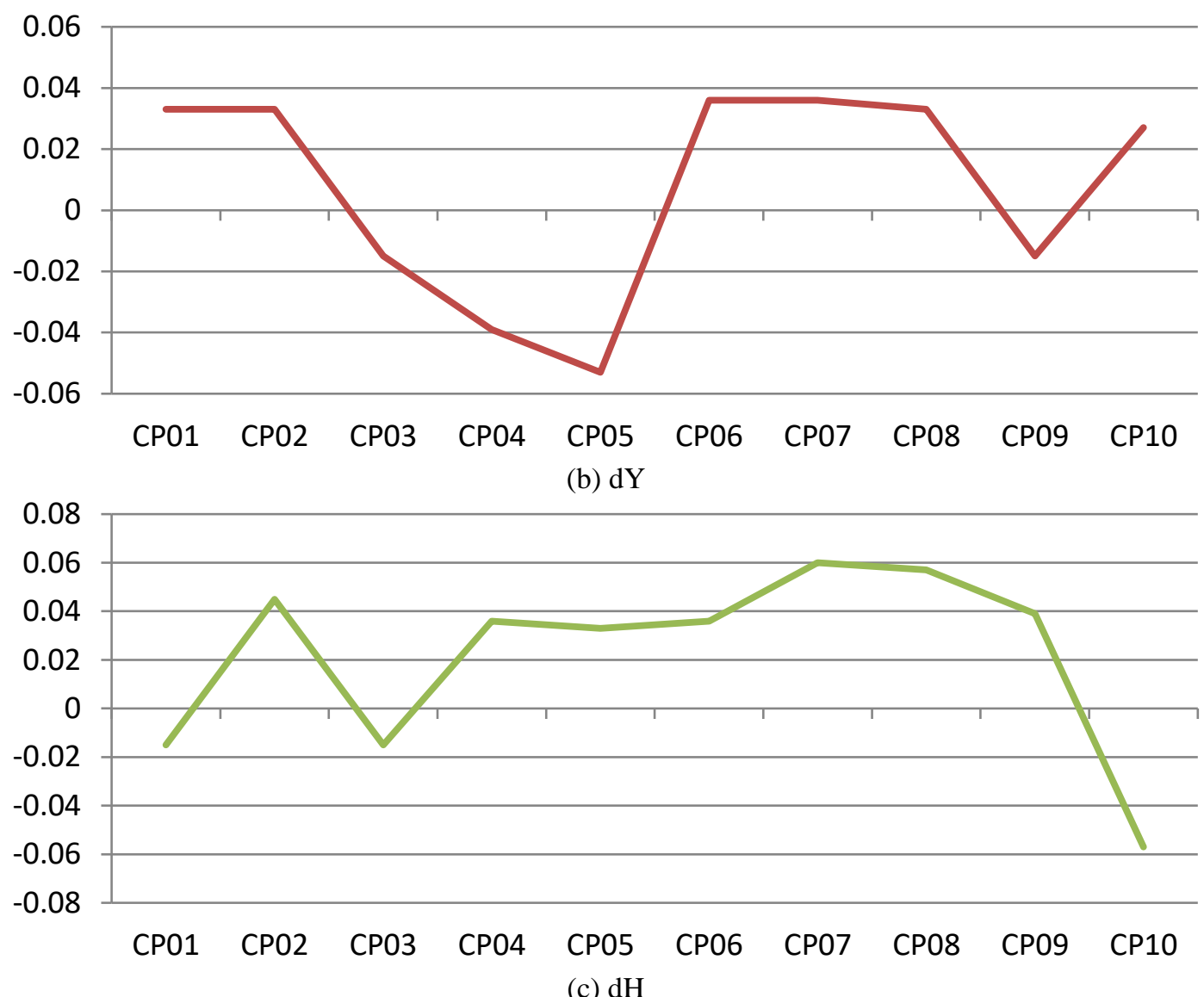

FiguRE. 5 Comparison of accuracy of check points

As a result of the accuracy evaluation compared to the check point, the maximum error was within $6 \mathrm{~cm}$ in the $\mathrm{N}, \mathrm{E}$, and $\mathrm{H}$ directions, and these results indicate the plane and elevation 
allowable accuracy of the scale 1: 1,000 digital map, suggesting the applicability of the method using the reference point performance and the laser scanning data. Table 1 shows the permissible accuracy for numerical mapping of Ministry of Land, Infrastructure and Transport.

Table 1. Permissible accuracy for numerical mapping[11]

\begin{tabular}{|c|c|c|}
\hline \multicolumn{3}{|c|}{ RMSE(m) } \\
\hline plane & Contour & Height \\
\hline 0.2 & 0.3 & 0.15 \\
\hline \multicolumn{3}{|c|}{ Maximum error $(\mathrm{m})$} \\
\hline plane & Contour & Height \\
\hline 0.4 & 0.6 & 0.3 \\
\hline
\end{tabular}

\section{Conclusion}

In this study, an accuracy analysis was performed on the new sensing technology of 3D laser scanning. Tunnel surveys using 3D laser scanners were conducted to assess their usefulness. The results of this study are as follows.

1. Results of accuracy evaluation compared with check points, the maximum error was within $6 \mathrm{~cm}$ in the $\mathrm{N}, \mathrm{E}$, and $\mathrm{H}$ directions, and this result indicates the plane and elevation acceptable accuracy of scale 1:1000 digital maps, and suggests the applicability of methods using reference point performance and laser scanning data.

2. Using scan data, continuous analysis of scan section shape as well as cross section analysis is possible.

3. Additional research can improve the accuracy of the feature registration method, thereby increasing the efficiency of tunnel investigation.

\section{Acknowledgements}

This research was supported by Basic Science Research Program through the National Research Foundation of Korea(NRF) funded by the Ministry of Science and ICT(No. NRF2018R1C1B6004021)

\section{References}

[1] S.H. Ju, S.H. Yoon, S.Y. Park, and J. Heo, "Simulation based Target Geometry Determination Method for Extrinsic Calibration of Multiple 2D Laser Scanning System”, Journal of the Korean Society of Surveying, Geodesy, Photogrammetry and Cartography, 36.6(2018): 443-450. DOI: 10.7848/ksgpc.2018.36.6.443

[2] S.Y. Kim, J.H. Yu, Y.G. Yu, and H.J. Lee, "atabase Enhancement for Development of Open-pit Mine Monitoring System in Open Source Environments”, Journal of the Korean Society of Surveying, Geodesy, Photogrammetry and Cartography, 34.1(2016): 21-32. DOI: 10.7848/ksgpc.2016.34.1.21

[3] S. Y. Soh, Y.J. Chun, Ambrose J. M. Itika, "A Study on Leaching and Solvent Extraction for the Recovery of Copper Ore for Small-Scale Mining in Tanzania", Journal of the Korea Academia-Industrial cooperation Society, 18.4(2018): 438-445.

[4] J.K. Park and K.W. Lee, "Analysis of the Status of Mine and Methods of Mine Geospatial Information Construction Technology for Systematic Mine Management", Journal of the Korea Academia-Industrial cooperation Society, 19.9(2018): 355-361. DOI: 10.5762/KAIS.2018.19.9.355 
[5] M.H. Kim and T.S Bae, "Preliminary Analysis of Network-RTK for Navigation", Journal of the Korean Society of Geodesy, Photogrammetry and Cartography, 33.5(2015): 343-351. DOI: 10.7848/ksgpc.2015.33.5.343

[6] J.K. Park, D.Y. Um, "Utilization Evaluation of Digital Surface Model by UAV for Reconnaissance Survey of Construction Project”, Journal of the Korea Academia-Industrial cooperation Society, 19.3(2018): 155-160. DOI: 10.5762/KAIS.2018.19.3.155

[7] K.D. Kim, S.H. Jung, K.H. Lee, Y.S. Choi, and M.S. Kim, "Mobile Mapping System Development Based on MEMS-INS for Measurement of Road Facility", Korean Society Of Surveying, Geodesy, Photogrammetry and Cartography, 36.2(2018): 75-84. DOI: 10.7848/ksgpc.2018.36.2.75

[8] K.W. Lee and J.K. Park, "Characteristic and Accuracy Analysis of Digital Elevation Data for 3D Spatial Modeling”, Journal of the Korea Academia-Industrial cooperation Society, 19.11(2018): 744-749. DOI: 10.5762/KAIS.2018.19.11.744

[9] J.K. Park and K.W. Lee, "Analysis of Geospatial Information Construction Efficiency by 3D Laser Scanner Integrated with Total Station", Journal of the Korea Academia-Industrial cooperation Society, 18.12(2017): 638-643. DOI: 10.5762/KAIS.2017.18.12.638

[10] Trimble Inc., (2019), https://www.trimble.com /

[11] National Law Information Center, Regulation of Technology Service enterprise, (2019), http://www.law.go.kr/

\section{Authors}

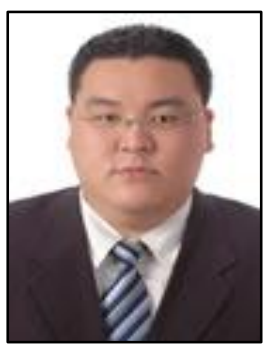

\section{Joon Kyu Park}

$\mathrm{He}$ is currently a Professor in Dept. of Civil Engineering at Seoil University. He received his B.S., M.S. and Ph.D. degree in Civil Engineering from Chungnam National University, Korea in 2001, 2003, 2008 , respectively. His research interests are in the areas of GPS, GeoSpatial Information Engineering. (jkpark@seoil.ac.kr)

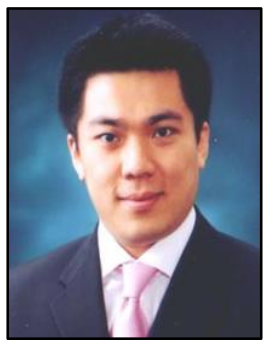

\section{Kap Yong Jung}

He is currently a Researcher in Dept. of Civil Engineering at Chungnam National University. He received his B.S., M.S. and Ph.D. degree in Civil Engineering from Chungnam National University, Korea in 2005, 2009,2013 , respectively. His research interests are in the areas of GeoSpatial Information Engineering. (jungjusa@ hanmail.net) 